\title{
DISSOCIATED PARALYSIS OF THE DIAPHRAGM FOLLOWING PHRENIC CRUSH AND PNEUMOPERITONEUM
}

\author{
BY \\ WALLACE FOX \\ British Legion Village Settlement, Preston Hall
}

In 1939 Douady and others reported for the first time dissociated paralysis of the diaphragm. It occurred in two patients during the course of phrenic alcoholization and pneumoperitoneum therapy for pulmonary tuberculosis. Since then no further cases have been found in the literature. The object of this paper is to report another case and to discuss the therapeutic and anatomical significance of this occurrence.

\section{CASE REPort}

The patient, a soldier, was diagnosed as having pulmonary tuberculosis in September, 1945, when he was aged 29. He had had a four-year history of lassitude and cough and had lost $1 \frac{1}{2}$ stone in weight. At the time of diagnosis he had a positive sputum, his blood sedimentation rate was $16 \mathrm{~mm}$. in the first hour (Westergren), and radiography showed considerable productive disease of the right upper lobe with extensive cavitation. In October, 1945, a right artificial pneumothorax was induced, but it was abandoned in November, 1945, due to the presence of extensive adhesions.

On Feb. 6, 1946, a right phrenic crush was performed under local anaesthesia. No accessory phrenics were found at the operation, and complete hemi-diaphragmatic paralysis was obtained. On April 24 screening showed that the diaphragm was recovering its function. The patient was admitted to Preston Hall on June 21, 1946, and at that time he had full diaphragmatic recovery. It was decided to repeat the right phrenic crush and to supplement it by a pneumoperitoneum as a preparation for thoracoplasty. So on July 24 the right phrenic crush was repeated, and again at operation no accessory phrenic nerves were found. Diaphragmatic paralysis with paradoxical movement resulted. On Aug. 2 a pneumoperitoneum was induced (Plate IXa).

Screening on Dec. 11, 1946, showed that a partial diaphragmatic recovery was occurring, and this recovery was of a striking nature. The anterior and medial part of the right hemi-diaphragm remained paralysed and showed slight paradoxical movement, whereas the lateral and posterior portion of the diaphragm was recovering its function, and within a few weeks displayed full active respiratory excursions.

On expiration the right hemi-diaphragm was shaped like a regular arc of a circle and there was only a slight point of demarcation between the paralysed and non-paralysed parts. On inspiration the appearance was strikingly altered. The posterior and lateral part descended actively, producing a very definite demarcation between the paralysed and non-paralysed parts of the diaphragm so that the regularity of contour was lost. This can be seen from the posteroanterior and lateral films taken on inspiration and expiration (Plate IX $b$ and Plate X $a, b$, and $c$ ).

The patient was observed for another six months before being transferred nearer home for his operation, and during this time the dissociated recovery of the diaphragm remained unaltered.

The diaphragmatic movement was a muscular contraction, and unlike the irregular movements occasionally produced by the presence of adhesions in the lower part of the pleural space. It differed from the denting of a recovering diaphragm which is occasionally seen in the presence of sub-diaphragmatic adhesions, and it was unlike the serrated appearance of some diaphragms in the presence of a pneumoperitoneum.

Anatomically, the immobile area of the diaphragm appeared to be the right leaf of the central tendon, the muscular fibres of the sternal origin, and the anterior muscle fibres of the costal origin. The area which had recovered its function comprised the posterior costal muscle fibres, the arcuate fibres, and the right crus. Hence, a true dissociated muscular paralysis of the diaphragm was present.

\section{Discussion}

Decker (1933) made passing mention of the fact that dissociated paralysis of the diaphragm could occur after phrenic crush. He stated: "In one case only a partial paralysis of the diaphragm was 

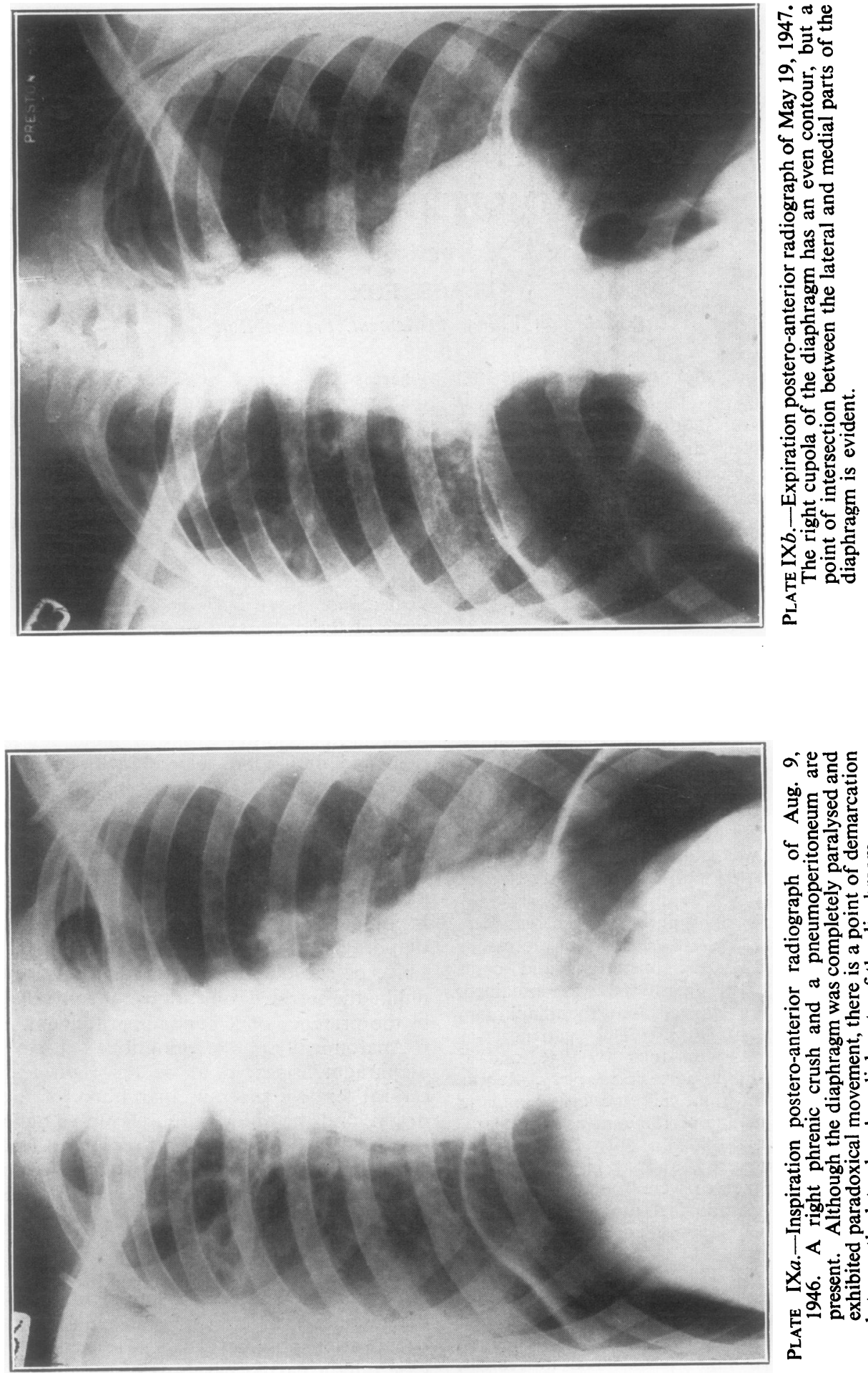

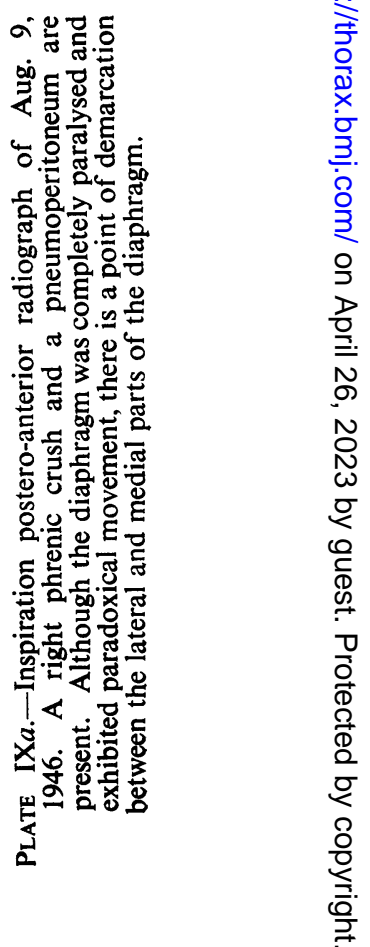




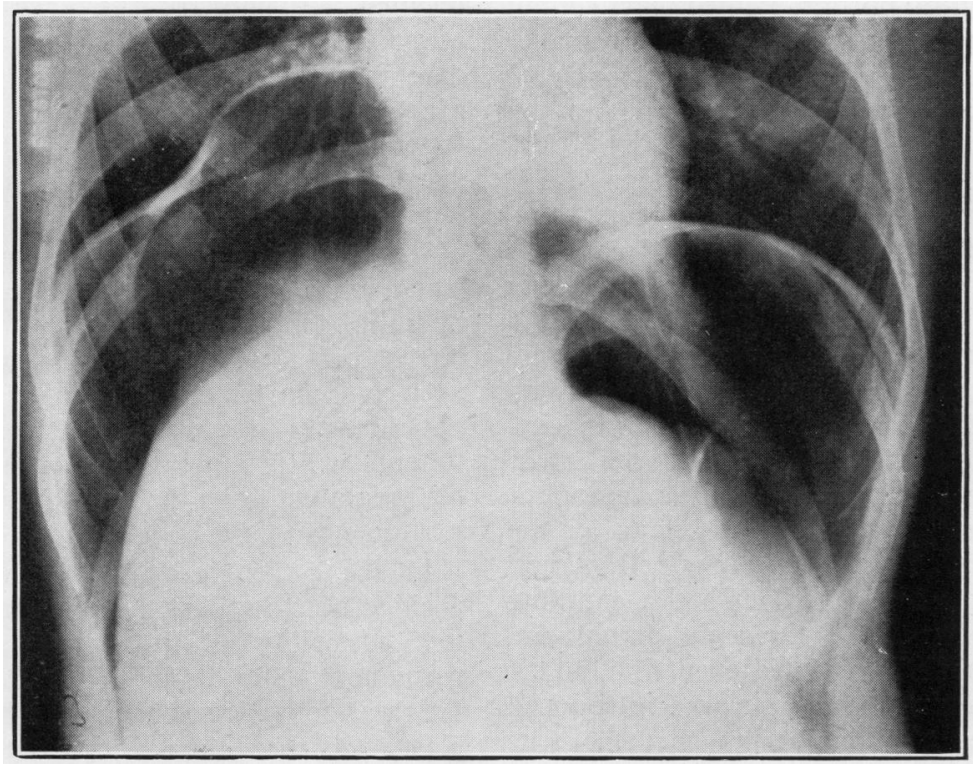

Plate Xa.-Inspiration radiograph of May 19, 1947. The diaphragm is now clearly divided into the medial paralysed portion and a lateral portion which has contracted actively.

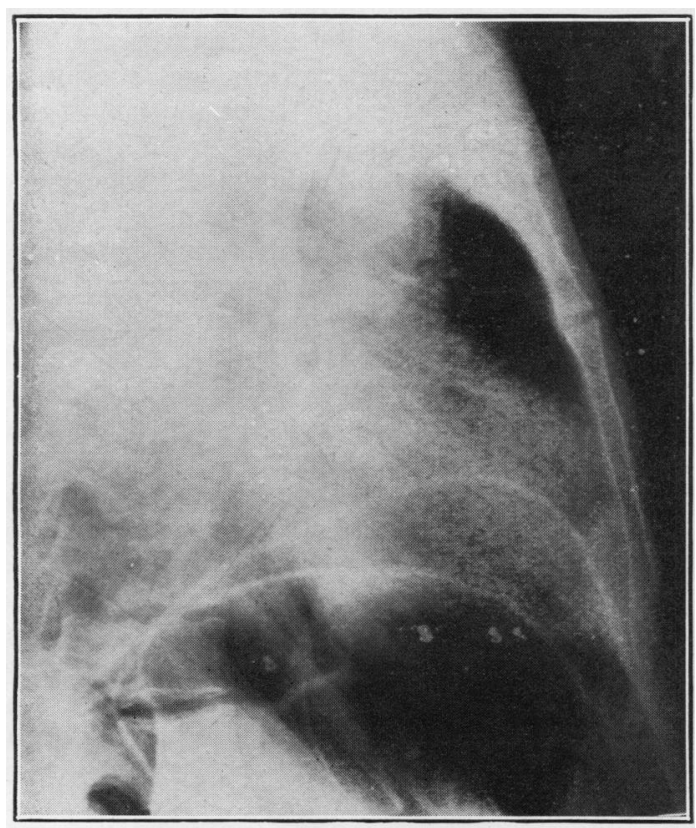

Plate X $b$.-Expiration right lateral radiograph of May 19, 1947. The contour of the right hemidiaphragm is seen to be smooth and regular, appearing to be a functional whole. The left cupola is also visible.

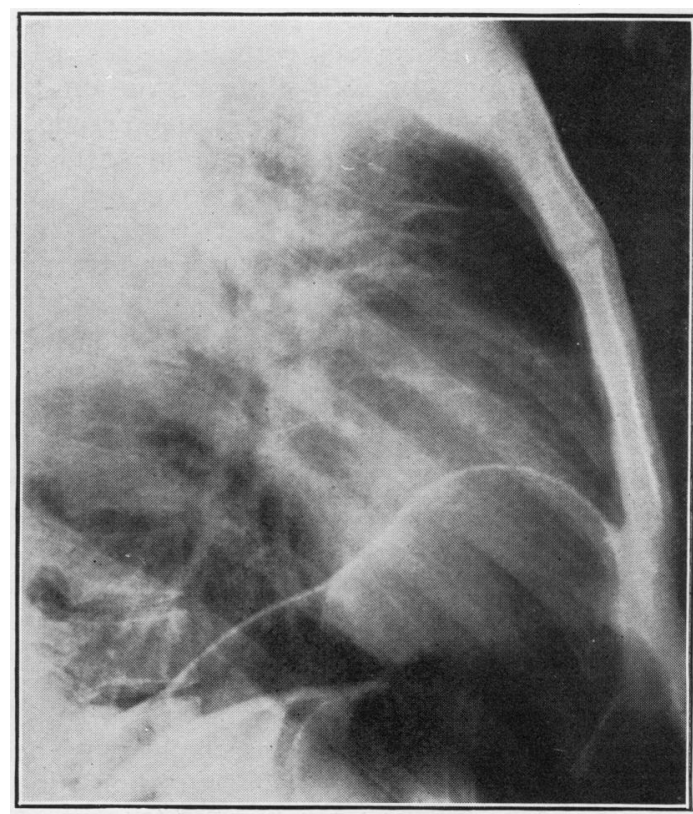

Plate X $c$.-Inspiration right lateral radiograph of May 19, 1947. It can be seen that, whereas the anterior part of the diaphragm has remained immobile, the posterior portion has descended actively on inspiration and the regularity of contour has been lost. 
obtained. The central portion seemed inactive, but the lateral peripheral portion functioned normally. A second operation was done, and then a sizable nerve, probably an accessory, was found on the scalenus medius muscle, overlapped by the scalenus anterior." Nehil and Alexander (1933) had a similar case in their series. Thus, it is occasionally possible to see a dissociated paralysis of the diaphragm in the absence of a pneumoperitoneum.

On the other hand, in the two cases quoted by Douady and others, the right hemi-diaphragm appeared pariesed after the phrenic crush and exhibited movement of diminished amplitude but of normal direction. It was only when a pneumoperitoneum was induced that it was possible to see the dissociated nature of the paralysis. It would appear that in these cases the liver had a damping effect on the movement of the right diaphragm, thus giving an overall diminished excursion, and it was only when a cushion of air was introduced between the liver and diaphragm that the true autonomy of the muscle was manifest, and the dissociated nature of the paralysis became evident. Hence, after phrenic crush, dissociated paralysis may become evident or may remain latent as an apparent diaphragmatic pariesis only to be recognized on the induction of a pneumoperitoneum.

Several observers have reported the return of diaphragmatic function after phrenic avulsion. In Morin's (1932) series of 343 cases diaphragmatic function returned in 2.5 per cent, and in Wirth and von Jaski's (1929) 185 cases 12.9 per cent had diaphragmatic recovery. This recovery was believed to be due to the presence of accessory phrenic nerves that were anatomically intact after the avulsions. It would appear likely that some of these cases were examples of latent dissociated paralysis, and that this occurrence is more frequent than has been realized in the past. In this respect, it is interesting to consider a case reported by John Alexander (1937). The patient had had a phrenic avulsion resulting in a complete diaphragmatic paralysis lasting for several years. In time almost complete recovery of respiratory movement was observed, and at a second operation on the phrenic nerve a small accessory phrenic was found and severed. Again complete paralysis resulted. Alexander comments: "This small root had presumably gradually assumed the functional load normally borne by the main phrenic trunk." At the initial phrenic avulsion the accessory phrenic nerve was probably traumatized, with a consequent interruption of conduction of nervous impulses, and so complete hemi-diaphragmatic paralysis resulted. Eventually the accessory phrenic recovered, and because of the contact of the diaphragm with the liver the whole diaphragm moved. But it is possible that if a pneumoperitoneum had been induced in this patient a dissociated paralysis would have been found.

It is important to recognize dissociated paralysis of the diaphragm. Most tuberculous lesions are situated posteriorly in the lung, and if after an operation on the phrenic nerve the posterior and lateral part of the diaphragm function normally, as in the reported cases of dissociated paralysis, the patient with a phrenic crush and pneumoperitoneum will probably derive no more benefit from his treatment than he would from the pneumoperitoneum alone.

In the patient reported in this paper it was decided not to repeat the phrenic crush in view of the possibility of producing a complete and permanent paralysis of the right hemi-diaphragm. This was considered undesirable because a thoracoplasty was the ultimate object of treatment.

The anatomical implications are of interest. Douady and others believe that in man the anterior and medial half of the diaphragm receives its nerve supply from cervical 3 and 4 and that the posterior and lateral portion is supplied by cervical 5. They were able to find an accessory phrenic nerve in one of their two patients, and division of this branch produced paralysis of the lateral and posterior part of the diaphragm. In addition experiments performed on the dog by Rodrigues and Carvalho (1939) support this opinion, for in one dog total resection of the right phrenic nerve produced a complete hemi-diaphragmatic paralysis, whereas resection of the superior root of the left phrenic nerve in the same animal produced a paralysis of the medial part of the left hemidiaphragm and the lateral portion continued to move normally.

In the patient of this report both the phrenic crush operations produced complete diaphragmatic paralysis. It was only when four months had elapsed after the second phrenic crush that the dissociated paralysis manifested itself. This fact, together with the apparent absence of accessory phrenics at both operations, would suggest that there were no accessory phrenics present in the patient and that all the phrenic nerve fibres lay in the one nerve trunk. The rate of regeneration of nerie fibres is very variable, but the fact that diaphragmatic recovery involved so exclusively the lateral and posterior part of the right hemidiaphragm suggests that the arrangement of nerve fibres in the phrenic nerve in this patient were such 
that at the second operation the fibres supplying this area of diaphragm suffered only physiological division, whereas the fibres to the anterior and medical part of the diaphragm suffered anatomical division. If the nerve fibres supplying the anterior and medial parts of the diaphragm occupied a circumferential position in the phrenic nerve, and the fibres to the lateral and posterior parts occupied the central part of the nerve, the latter are less likely to suffer such severe damage as the circumferential fibres. Furthermore, the presence of perineural fibrosis as a result of the first phrenic crush would also protect the central fibres of the nerve at a second operation.

\section{SUMMARY}

A case of dissociated paralysis of the diaphragm occurring in the course of treatment of pulmonary tuberculosis by phrenic crush and pneumoperitoneum therapy is described and illustrated by radiographs.
The fact that this condition may not be detected is stressed, and the therapeutic and anatomical significance is discussed.

I am indebted to Dr. F. Temple Clive, Medical Superintendent of Preston Hall, for his permission to publish the case report, and to Dr. F. R. G. Heaf, Honorary Consulting Medical Director, and Dr. Simon Behrman for their advice during the preparation of the paper.

\section{REFERENCES}

Alexander, John (1937). "Collapse Therapy of Pulmonary Tuberculosis." Charles C. Thomas, Springfield. P. 148.

Decker, H. R. (1933). J. thorac. Surg., 2, 545.

Douady, Lardanchet, and Venator (1939). Arch. méd.-chir. Appar. resp., 14, 275.

Morin, J. (1932). Schweiz. med. Wschr., 13, 721.

Nehil, L. W., and Alexander, John (1933). J. thorac. Surg., 2, 549.

Rodrigues, A., and Carvalho, R. (1939). Arch. méd.chir. A ppar. resp., 14, 27.

Wirth, A.. and von Jaski, Kohn (1929). Beitr. klin. Tuberk., 73, 1. 